\title{
eJRIEPS
}

Ejournal de la recherche sur l'intervention en éducation physique et sport

32 | 2014

Varia

\section{Au football, la règle du hors-jeu a-t-elle encore un sens?}

\section{Patrick Marle et Eric Duprat}

\section{(2) OpenEdition}

\section{Journals}

Édition électronique

URL : http://journals.openedition.org/ejrieps/2097

DOI : 10.4000/ejrieps.2097

ISSN : 2105-0821

Éditeur

ELLIADD

\section{Référence électronique}

Patrick Marle et Eric Duprat, « Au football, la règle du hors-jeu a-t-elle encore un sens ? », eJRIEPS [En ligne], 32 | 2014, mis en ligne le 01 avril 2014, consulté le 03 octobre 2019. URL : http:// journals.openedition.org/ejrieps/2097; DOI : 10.4000/ejrieps.2097

\section{(c) (1)}

La revue eJRIEPS est mise à disposition selon les termes de la Creative Commons Attribution 4.0 International License. 
eJRIEPS 32 avril 2014

Au football, la règle du hors-jeu a-t-elle encore un sens ?

Patrick Marle* \& Eric Duprat ${ }^{\star *}$

* Professeur honoraire de l'Université de Franche-Comté

** Département STAPS. Université d'Evry

\section{Résumé}

L'objet de cet article est de proposer un point de vue sur la difficulté à appliquer la règle $d u$ hors-jeu au football. En soi, il ne constitue pas une recherche, mais une réflexion avec comme question centrale "Comment favoriser le jeu en football ? ". La première section examine l'histoire de cette règle. La deuxième section est centrée plus particulièrement sur son évolution actuelle et son application et enfin, la troisième discute de sa pertinence et de sa légitimité dans le football professionnel.

Mots clés : football, règles, hors-jeu, pertinence, sens.

Chaque pratique sportive collective possède des origines déterminées et s'accompagne d'une philosophie du jeu qui lui est propre. Aussi, intervenir sur les règles premières d'une pratique de référence provoque des transformations qui en font une autre activité : le football gaélique, le football australien et le football américain en sont des exemples pertinents avec comme parent commun le football du XIX ème siècle. Dans l'histoire du football, son évolution a toujours précédé sa codification (ou réglementation). On a commencé par jouer, à pratiquer les jeux avant de, peu à peu, stabiliser les règles et résoudre les conflits entre les différentes écoles. II en est de même du statut et du rôle de l'arbitrage qui petit à petit se sont imposés comme indispensables. Au départ, jouer sans arbitre était la norme. Puis, on est passé progressivement d'un arbitre conseil auprès des capitaines des deux équipes, qui devaient trancher les différents, à un rôle de censeur "muni d'un sifflet et maître absolu de la partie " (Deleplace, 1966, p.338). C'est-à-dire une personne qui est positionnée comme censeur et juge rigoureux vis-à-vis de la prestation d'autrui, avec tous les écueils que cela peut comporter. Dans les sports collectifs, il est maintenant indispensable, d'avoir un ou plusieurs arbitres qui doivent rester neutres et calmes pendant toute la partie, tout en observant attentivement les situations afin de pouvoir rendre un jugement objectif. L'arbitre participe au contrôle du jeu et réprime les infractions. Néanmoins, sa position est difficile, peu enviée et souvent 


\section{eJRIEPS 32 avril 2014}

mal reconnue. Kssis \& Couvidou (2013) soulignent que l'institutionnalisation des règles des jeux de grand terrain, à la fin du dix-neuvième siècle, a eu pour principal écueil la tendance à déresponsabiliser les joueurs au profit d'une autorité supérieure, seule garante de la probité et de la régularité des matchs. Maintenant, l'arbitre impose ses décisions alors qu'avant il n'intervenait qu'à la demande des équipes qui s'estimaient désavantagées. Cette conception d'un véritable «deus ex machina a transformé le joueur d'un rôle actif dans le contrôle du jeu à un rôle de passif obéissant car il peut continuer tant que ce n'est pas sifflé ! Ce rapport actif / obéissant a contribué à développer des stratégies de dissimulation ou de simulation voire de triche. On est libre de faire ce que l'on veut tant qu'on ne se fait pas prendre : credo bien moderne de l'individualisme contemporain (Lipovetsky, 1983). Alors, ce qui prévaut dans la conscience du joueur n'est pas de se conduire de manière correcte mais ce que sanctionne ou non l'homme en noir (Andrieu, 2013). Cependant, cet arbitre est le plus souvent traité en bouc émissaire. Car, qui n'a pas vécu un match à côté d'un supporter exalté passant son temps à faire des commentaires désobligeants sur la façon d'arbitrer. En général, ce spectateur enrubanné des attributs et couleurs de son club ne connaît que superficiellement les règles, a peu pratiqué la discipline, mais possède des réponses à tout.

Dans certains jeux à effectif réduit et certaines fédérations sportives, l'application des règles est fondée sur l'auto-arbitrage, comme dans l'ultimate. Les fautes, si elles existent, sont en effet indiquées par les joueurs eux-mêmes. L'auto-arbitrage ne va-t-il pas alors plus loin que le jeu lui-même ? En effet, voir et reconnaître ses erreurs, c'est prendre ses responsabilités et ne pas rejeter la décision sur l'autre. Tout le monde y gagne car les joueurs se comportent plus sereinement. Les clubs ont des joueurs qui s'investissent dans la vie et les obligations du groupe. Les spectateurs peuvent se consacrer au seul plaisir de regarder une pratique sportive sans s'occuper de l'arbitre et sans entendre de reproche à son encontre. Mais, cela est-il compatible avec le sport professionnel et le sport spectacle où l'enjeu prime sur le jeu ? Ici, il faut garantir la rentabilité et «l'honnêteté du score ». Aussi, dans le passé et l'avenir économique du football, arbitre et professionnalisme sont indissociablement liés. Concernant la stricte application des règles, l'introduction de la vidéo au rugby de haut niveau n'a pas apporté non plus la solution miracle espérée. Parfois le doute persiste encore malgré les images au ralenti et le risque d'une sur-utilisation est bien réel. Par ses excès, les limites du recours à la technologie en lieu et place d'une certaine éthique du jeu déresponsabilisent les acteurs. Cet arbitrage vidéo, idée séduisante a priori, a bien montré par ses excès (temps de jeu 


\section{eJRIEPS 32 avril 2014}

hachés par des arrêts plus ou moins intempestifs) les limites du recours à la technologie en lieu et place d'une certaine éthique à propos du jeu. Alors, la question de la règle et de son évolution, à la fois comme contrainte pour le joueur mais aussi comme source de développement et de progrès quand on joue avec celle-ci, se pose. En football, dire que l'activité des joueurs dépend directement des règles qui régissent le jeu et de leur compréhension apparaît comme une évidence. L'évolution des règles sportives témoigne, aussi, de la dynamique inventive de l'homme.

A partir de tous ces constats, nous allons examiner, dans cet article, le règlement du football, son évolution et l'application courante qui en est faite et poser, en particulier, la question de la nécessité de la règle du hors-jeu compte tenu de l'évolution des rapports d'opposition.

\section{Un peu d'histoire}

Les règles sportives sont souvent pensées comme intemporelles et immuables, néanmoins elles vivent et se transforment au cours du temps (Léziart, Gabagno, Loquet, \& Trohel, 2012). Depuis le XIX ème siècle, les conditions de l'affrontement ont bien changé précédant ou accompagnant l'évolution du jeu et de ses règles. "Les jeux de ballon sont au nombre des plus anciens exercices de plein air que pratique l'humanité. II n'en est pas de plus amusants, de plus animés, de plus propres à développer l'agilité, la force musculaire, le souffle, la vitesse, l'esprit d'à propos. Dans le jeu de l'Association (football), un joueur avisé ne perdra jamais de vue le ballon tout en surveillant sans cesse l'ennemi pour voir s'il ne laisse pas quelque trou dans ses rangs et quelque point faible dans sa défense. II s'exercera, sans relâche à bien chasser le projectile. A cet effet l'adresse et le savoir-faire importent encore plus que la vitesse (...), et il est incontestable que, dans l'art tout spécial du "chasser" (conduite de balle), un oeil prompt, un jugement sûr, un esprit rompu au calcul de chance valent mieux que tout» (Daryl, 1894). En effet, s'il était possible de voyager dans le temps et l'espace, de s'installer sur la ligne de touche d'un match joué vers le milieu du XIX ème siècle, le style de jeu nous surprendrait inévitablement du fait de la rareté des échanges de balle. Chaque attaquant prenait alors le ballon et le conduisait jusqu'à ce qu'il le perde ou tire aux buts. Ainsi, chaque attaquant faisait sa partie sans se préoccuper de ses coéquipiers, " cherchant à conquérir quelques lauriers par son adresse comme dribbleur, chassant le ballon devant lui pour le piloter par d'habiles manoeuvres à travers les vides dans les rangs ennemis, lui faisant faire maints détours pour éviter la défense du camp opposé et arriver jusqu'au but. Toute l'équipe suivait. La seule combinaison admise alors consistait à soutenir un coéquipier dans 


\section{eJRIEPS 32 avril 2014}

l'espoir, s'il venait à perdre le ballon, de le lui "chiper" et de faire à son tour une jolie exhibition de dribbling » (Tunmer - Fraysse, 1904).

On pourrait assister également à un événement insolite et inattendu pour des spectateurs modernes, les capitaines des deux équipes discutant âprement le règlement à appliquer dans la rencontre qui allait se dérouler. Par exemple, une des discussions qui revenait souvent consistait à savoir si l'on autorisait ou non les coups de pieds dans les tibias ; tout un programme (Gréhaigne, Meunier, \& Vigneron, 2007). A cette époque de rugby et football non différenciés, le terrain devait avoir une longueur de 200 yards (180 m environ) et une largeur maximale de 100 yards (90 m environ), les buts étaient délimités par deux montants verticaux placés à une distance de 8 yards $(7,32 \mathrm{~m})$ l'un de l'autre sans barre transversale. Le but était marqué lorsque le ballon passait entre les poteaux, à n'importe quelle hauteur, ou si un joueur de l'équipe attaquante touchait le ballon le premier derrière la ligne de but adverse (l'en-but au rugby). Les attaquants avaient droit à un coup franc tiré seulement en direction du but à partir d'un point situé à 15 yards (14 $\mathrm{m}$ ) de la ligne de but. Sachant que l'on pouvait soit marquer directement soit toucher derrière la ligne, il arrivait souvent que plusieurs joueurs soient très avancés, prêts à aplatir le ballon dès qu'il avait franchi la ligne. II s'en suivait des regroupements peu propices à favoriser le jeu. En conséquence le jeu devenant impossible, il fut institué en 1866 la loi du hors-jeu à trois joueurs selon laquelle, pour ne pas être «hors-jeu», l'attaquant le plus avancé devait avoir au moins trois adversaires entre lui et le but opposé. Règle importante qui ne devait subir que peu de modifications importantes dans le temps.

Au football ou au rugby, les lois sur le hors-jeu limitent et cadrent la circulation des joueurs sur le terrain. Au handball, devant chaque but, il existe une surface interdite aux joueurs sauf au gardien : c'est un demi-cercle de $6 \mathrm{~m}$ de rayon (la zone) qui est quand même largement utilisé dans les airs. Au basket-ball, devant le panier, se trouve une raquette où l'on ne peut pas stationner plus de trois secondes. Enfin, au hockey sur glace, on retrouve, dans le tiers avant de la patinoire, une zone tenant toute la largeur où la rondelle doit pénétrer avant tout attaquant lors d'une montée offensive. Ces lois sur le hors-jeu ou sur les zones interdites de façon permanente ou temporaire, visent, en fait, à établir ou rétablir un équilibre entre les droits de l'attaque et ceux de la défense (Gréhaigne \& Marle, 2013). Cela correspond également à un aspect très moral à savoir que « dans le jeu, nul ne peut s'approprier un avantage immérité ». Le but de l'ensemble de ces règles est le même dans tous les sports collectifs. C'est d'empêcher les avants «piquets » de se positionner à proximité du but de l'autre équipe et d'attendre l'opportunité de marquer. En ce qui concerne plus particulièrement le rugby, Deleplace 
eJRIEPS 32 avril 2014

(1966, p. 342) remarque que « l'idée de départ qui amène la règle du hors-jeu est le parti pris athlétique ». Les gains de terrain que réalise le ballon tantôt vers un en-but, tantôt vers l'autre, doivent résulter des actions d'un ou plusieurs joueurs. Le rugby veut que ce soit les hommes qui courent, et non le ballon à leur place. Le mouvement du ballon par rapport à la profondeur du terrain ne doit être que le fruit du mouvement des hommes selon cette dimension.

\section{1. D’abord un jeu d'écolier}

Dans les années 1820, le football moderne a commencé à prendre forme dans les écoles publiques (Public School) d'Angleterre. Les instituteurs ont reconnu que le jeu développait la santé et la force et pouvait être « civilisé ». Dans les écoles, y compris Eton, Harrow, Rugby et Westminster, on a commencé à jouer à des jeux récréatifs avec une « balle » qui était plate à la fois sur le dessus et sur le dessous. Chaque école développa ses propres règles et définit son propre terrain. Avant que ces écoles aient commencé à organiser le sport, les matchs étaient joués dans des espaces ouverts sans limites. Le 26 octobre 1863, des représentants de onze écoles et clubs se réunirent à la Taverne des «Francs Maçons » pour arrêter les règles officielles du jeu. Un club, Blackheath, s'est finalement retiré des débats après que le groupe ait décidé d'interdire de courir en tenant le ballon et de donner des coups de pied dans les tibias des adversaires. Les dix représentants restants formèrent la Football Association. Lorsque cette décision fut prise, la séparation entre les jeux collectifs qui allaient devenir le football et le rugby était devenue quasi officielle. Le 8 décembre 1863, le Football Association et le Football Rugby furent finalement divisés en deux organisations différentes. Les membres de la Football Association publièrent les quatorze premières lois du Jeu en décembre 1863.

De façon surprenante, ces premières lois ne contenaient aucune mention de fautes, de pénalités ou d'arbitres. Malgré son héritage violent, le football à cette époque était considéré comme un sport de gentlemen : des messieurs, pensait-on, ne seraient pas tenter de se salir pour obtenir un avantage. En effet, à l'origine, les capitaines d'équipe se consultaient pour résoudre n'importe quel conflit sur le terrain. Finalement, ce rôle a été délégué à un arbitre. Chaque équipe amenait son propre arbitre permettant aux capitaines des équipes de se concentrer sur le jeu. Plus tard, l'arbitre, un troisième officiel « neutre » a été ajouté. Cet arbitre était « consulté » si les arbitres ne pouvaient pas résoudre un conflit. Petit à petit, cet arbitre a pris place sur le terrain et en 1891, les arbitres fournis par les équipes sont devenus des assistants (arbitres de ligne). Aujourd'hui encore, dans beaucoup de matchs de football amateur, chaque côté fournit toujours un assistant à l'arbitre central pour aider celui-ci. 


\section{eJRIEPS 32 avril 2014}

1. 2. L'origine de la règle du hors-jeu (loi 11) : limiter l'espace

La nécessité d'une loi sur le « hors-jeu » remonte aux premières années du jeu générique de football. Un joueur était « hors de son côté de jeu » s'il se tenait face à la balle entre le ballon et le but de l'adversaire. A cette époque plusieurs groupements cohabitaient. Pour l'Association de Sheffield, cela n'avait aucune importance ! II n'y avait pas de règle du hors-jeu et les joueurs dits « tireurs » étaient positionnés en permanence près ou devant le but adverse.

Mais, la plupart des dirigeants et des joueurs qui ont rédigé les règlements successifs, au milieu du XIXe siècle, avaient été formés avec l'idée de «positionner » tous les joueurs « derrière » la balle, interdisant la passe en avant. On conservait ainsi l'objectif de base de l'époque : pour progresser vers le but opposé on devait avancer en dribble avec le ballon ou à l'aide d'une mêlée. On se rendit compte bien vite que pour assurer le développement du football association il fallait assurer la libre circulation du ballon, il fallut donc autoriser la passe vers l'avant, augmentant ainsi la nécessité d'une loi sur le horsjeu. Les règles de Cambridge de 1848 avaient opté pour trois adversaires entre un avant et le but opposé pour être en jeu. Toutefois, les règles Uppingham de 1862 continuèrent de prohiber strictement la passe en avant et celles-ci furent adoptées, temporairement, par toute la famille du football association.

Comme le football s'était développé dans les années 1870, la passe en avant et la loi du hors-jeu ont continué à être la cause de disputes entre les clubs. Sheffield se débarrassa des «tireurs postés » en modifiant ses lois afin qu'un membre de l'équipe en défensive soit toujours entre l'avant et le but adverse. La Fédération de Football Association adopta l'idée de l'école de Cambridge avec trois joueurs, et finalement Sheffield se mit en conformité avec la norme des trois joueurs qui fut la règle jusqu'en 1925.

\section{3. L'essence du jeu}

Le football et les sports collectifs sont basés sur des problèmes qui, dans une certaine mesure, peuvent apparaître arbitraires. Pour Brackenrigde, (1979) c'est une lutte pour la conquête d'un territoire avec un ensemble de règles (paramètres structuraux) qui incluent des stratégies significatives (cognition) et des aspects techniques (action) où l'anticipation / coïncidence est de la plus grande importance. Le règlement identifie les problèmes et garantit pour l'ensemble des équipes ou des individus l'égalité des chances. La caractéristique principale de l'analyse de Brackenbridge est de se focaliser sur les règles qui fournissent une structure pour le jeu étant donné qu'elles spécifient clairement la nature du problème posé par la pratique et restreignent les moyens disponibles pour les joueurs afin de répondre aux problèmes posés. Les moyens de résoudre ces problèmes 


\section{eJRIEPS 32 avril 2014}

ne sont pas toujours évidents et efficaces. Ainsi, il serait certainement plus facile de marquer au football si l'on pouvait s'aider des mains, mais ce ne serait plus du football. Les règles du jeu ont été établies dans le but de fournir une structure qui permette au jeu et aux émotions qui l'entourent d'être répétées et communiquées aux autres. Excepté au rugby, il est patent que ces règles sont inspirées par une conception des rapports humains excluant la toute primauté de la force physique sur autrui.

Bien sûr, les jeux sportifs collectifs ont évolué à travers les années mais leurs principales caractéristiques restent aisément identifiables. Ils coïncident tous par la résolution de problèmes dans laquelle un des participants tente de prendre l'ascendant sur un autre. Tous les jeux sportifs collectifs sont caractérisés par des règles qui fournissent une structure définissant le problème à résoudre (cf. aire de jeu, équipement, nombre de joueurs, temps accordés). A cet effet, des stratégies, des tactiques sont utilisées ce qui requiert de la technique pour les mettre en œuvre.

Afin d'identifier un jeu, nous parlerons de règles premières qui garantissent que le problème du jeu sera toujours le même. Les règles premières ou constitutives ne définissent pas le but du jeu (cf. la finalité : marquer un but de plus que l'adversaire), elles concernent les moyens utilisés pour atteindre cette finalité. On pourrait dire que l'on peut atteindre cette finalité en employant d'autres moyens que « jouer le jeu ». Ainsi, les règles premières, puisqu'elles décrivent les moyens, fournissent au jeu ses caractères essentiels : les règles premières font que le rugby n'est pas le football ou le hand-ball. Selon Deleplace $(1966$; 1979) la logique interne du jeu serait le produit de l'interaction continue, au cours du développement historique du jeu, entre les principales conventions du règlement et l'évolution des solutions pratiques apportées par les joueurs en relation avec les progrès réalisés dans le développement des habiletés tactiques, techniques et de la condition physique. Pour une pratique de référence donnée, le règlement conditionne donc, en grande partie, les rapports d'opposition car il détermine la marge de manœuvre à l'intérieur des contraintes qu'il impose. Il est organisé autour d'un noyau central stable. En sport collectif, les règles premières reposent sur :

- la marque, ses modalités particulières relatives aux spécificités des cibles du sport concerné. C'est en effet à partir des caractéristiques de l'espace de jeu, de la ou des cibles, et des habiletés nécessaires pour marquer, que dépendent la logique du règlement et les logiques d'actions qui régulent les rapports d'opposition ;

- les droits des joueurs en attaque et en défense, dans le cadre de l'utilisation du ballon et de son appropriation, qui découlent de la logique de la marque et la complètent dans le respect de la nécessaire égalité des chances ; 
eJRIEPS 32 avril 2014

- le degré de liberté d'action sur la balle qui permet de faire vivre de manière spécifique le jeu en favorisant la continuité des mouvements et en «libérant » les remises en jeu. Ou encore, la loi 11 (hors-jeu) en football qui impose l'utilisation de l'espace à certaines conditions ;

- les modalités d'engagement physique qui assurent le respect du "parti pris athlétique" sportif dans le cadre des trois règles précédentes.

Ces règles que l'on nomme aussi fondamentales ou constitutives sont enrichies par des règles complémentaires ou règles secondaires qui évoluent pour tenter de s'adapter aux transformations des pratiques tout en conservant "l'esprit du jeu". Nous allons envisager cette évolution de l'ensemble des règles au football et plus spécialement de la loi 11 sur le hors-jeu

\section{L'évolution du hors-jeu au football}

En sport collectif, dire que les stratégies et les tactiques utilisées par les joueurs sont formatées directement par les règles qui régissent le jeu est patent. Néanmoins, il faut pourtant constater que le jeu constitue une réalité à part, à la fois dépendante et indépendante du règlement. Le jeu lui-même possède une vie propre, il se transforme petit à petit avec la pratique des joueurs au fil du temps. Dans ce mouvement d'évolution propre au jeu lui-même, il s'ensuit souvent une complexification du jeu, preuve à la fois de la fécondité des règles et des capacités d'imagination et d'adaptation des joueurs. Parfois, ces mutations vont jusqu'à entrer en contradiction avec les règles existantes, à s'opposer à telle ou telle partie de ces règles. L'apparition de telles contradictions, qui viennent briser le lien entre règlement et esprit du jeu, entraîne la nécessité d'une remise en ordre qui ne peut revêtir que deux aspects. Soit on interdit la nouveauté qui a fait naître la contradiction, soit on vise une évolution du règlement intégrant la nouveauté amenée par la vie du jeu, quitte à ouvrir encore à partir de là de nouvelles perspectives d'évolution du jeu.

Par exemple, il apparaît une différence notable entre la règle originelle et la règle actuelle. $\mathrm{Au}$ tout début le hors-jeu. A l'époque, le hors-jeu se jugeait à l'arrivée de la balle. Cette caractéristique permettait aux défenseurs de manœuvrer facilement pour 'mettre' les avants adverses hors-jeu, mais était plus facile à arbitrer. Maintenant, c'est au moment où le ballon est joué que se juge le hors-jeu. La difficulté pour l'arbitre assistant (de touche) est d'évaluer, dans le même temps, le départ du ballon et la position de l'attaquant le plus avancé. L'expertise des arbitres, nécessite de prendre des décisions dans des contextes de plus en plus complexes et contraints. Quand immédiatement après l'action, la 


\section{eJRIEPS 32 avril 2014}

télévision repasse dix fois, au ralenti, les éventuelles erreurs d'appréciation, cela nuit à leur crédibilité. II est à noter que Dosseville et Laborde (2011) indiquent que certains facteurs psychologiques, physiologiques et émotionnels améliorent, déterminent ou influencent leurs prises de décisions. Les résultats de leurs expériences ont montré que la fréquence cardiaque et la vitesse de déplacement de l'arbitre variaient significativement selon le type de décision concerné, mais également entre la perception de l'action et les décisions qu'il avait à prendre.

Pour en revenir à la règle du hors-jeu, l'essentiel de la rédaction précédente datant de 1925 spécifiait : « est en position de hors-jeu, tout joueur qui est plus rapproché de la ligne de but adverse que le ballon au moment où celui-ci est joué » et puis était énuméré un certain nombre d'exceptions qui dispensaient de la sanction. Maintenant, la règle dit : « II n'y a pas d'infraction de hors-jeu lorsque le joueur :

- se trouve à la même hauteur que l'avant-dernier adversaire (ou les deux derniers adversaires);

- se trouve derrière le ballon lors de la passe ou sur la même ligne ;

- reçoit le ballon d'un joueur de l'équipe adverse qui en avait le contrôle parfait. Ainsi, une passe faite par un joueur de l'équipe $A$ qui dévie sur le gardien ou un défenseur de l'équipe $B$ et qui arrive à un joueur de l'équipe $A$ en position de hors-jeu au moment où la passe est faite mène à un joueur hors-jeu. En revanche, si le gardien, balle à la main, rate son dégagement et envoie la balle sur le joueur hors-jeu, il n'y aura pas de faute.

- reçoit le ballon sur une remise en jeu du type sortie du terrain (corner, touche, coup de pied de but) ;

- se trouve dans sa propre moitié de terrain de jeu ou sur la ligne médiane.

Il y a faute si un joueur en position de hors-jeu intervient dans le jeu, soit en touchant le ballon, soit en gênant ou influençant un autre joueur ».

La grande différence est actuellement, que le jeu permet aux joueurs d'être n'importe où sur le terrain sans être automatiquement sanctionné d'être hors-jeu. Pour qu'un joueur puisse être pénalisé pour hors-jeu, il doit être impliqué activement dans l'action de jeu. Si le joueur reste sur place et attend que le jeu le rattrape, alors c'est conforme au nouveau règlement. S'il reste l'autre côté du terrain loin de la balle, là encore il n'y a pas de horsjeu. Pour faire "simple" la position de hors-jeu n'est plus une infraction en elle-même. C'est aux arbitres assistants de prendre leurs responsabilités et laissant réapparaître les problèmes d'interprétation et les discussions. En fait, la nouvelle rédaction n'a pas fondamentalement changé la donne. Ce n'est plus une loi d'exception mais le problème d'appréciation persiste. 


\section{eJRIEPS 32 avril 2014}

\section{Discussion}

Teneze (2011) a bien montré dans son étude de la loi 11 que l'évolution du hors-jeu a provoqué une transformation de la logique interne du jeu : on est passé d'une ligne d'affrontement, plutôt violente, à une libre circulation des joueurs sur presque tout le terrain. Cette évolution peut être illustrée par le changement de repères concernant la ligne flottante : d'abord le ballon en 1863, puis l'antépénultième défenseur vers 1870, et enfin l'avant dernier défenseur en 1925. En conséquence, cette transformation se caractérise par une diminution progressive des possibilités de hors-jeu pour les joueurs, entraînant en conséquence une augmentation de leur espace d'interaction dans le jeu en mouvement. En 2004, une décision de l'International Board met quasiment fin, en pratique, au fameux hors-jeu de position. Désormais, "prendre une part active au jeu en influençant l'adversaire » signifie perturber ou empêcher un défenseur de jouer ou d'être en mesure de jouer le ballon. Un joueur ne doit pas faire un geste ou un mouvement en se trouvant sur la trajectoire du ballon pour tromper ou désorienter un adversaire. II faut donc qu'un joueur en position de hors-jeu et qui ne touche pas le ballon entrave explicitement les mouvements ou la vue des défenseurs (gardien compris) pour que sa position soit sanctionnée. En d'autres termes, cela revient à faire disparaître la quasitotalité des hors-jeu de position. Bien sûr, quelques éléments et interprétations sont fournies aux arbitres et à leurs assistants pour les aider à se forger un jugement sur cette nouvelle signification de la Loi 11. Néanmoins des divergences d'interprétation demeurent. La confrontation de plusieurs arbitres experts à la lecture de vidéo où figuraient des hors-jeu a révélé des prises de décisions disparates (Fuss, Subic, \& Ujihashi, 2007).

II apparaît évident que dans l'espace de jeu direct un attaquant plus rapproché de la ligne de but influence toujours le jeu. A titre d'exemple présenté dans la figure 2, le joueur hors du jeu influence-t-il le jeu des deux défenseurs entourés de rouge ? La réponse est clairement oui : des adversaires dans le dos des défenseurs sont toujours un problème. Où commence l'influence sur le jeu ? Première affirmation : un joueur en position de horsjeu influence toujours, même si c'est de façon minimale, la configuration momentanée du jeu. Revenir d'une position de hors-jeu constitue également un trouble certain dans les rapports d'opposition. Un sport collectif comme le rugby a réglé le problème car, dans le jeu en mouvement, il n'y a pas de hors-jeu puisque la passe en avant est interdite, sauf sur les coups de pied afin de préserver le «parti pris athlétique ». Alors, hors-jeu, hors-jeu de position, l'arbitre siffle ou on ne siffle pas. Est-ce un bon moyen pour avantager l'attaque? 


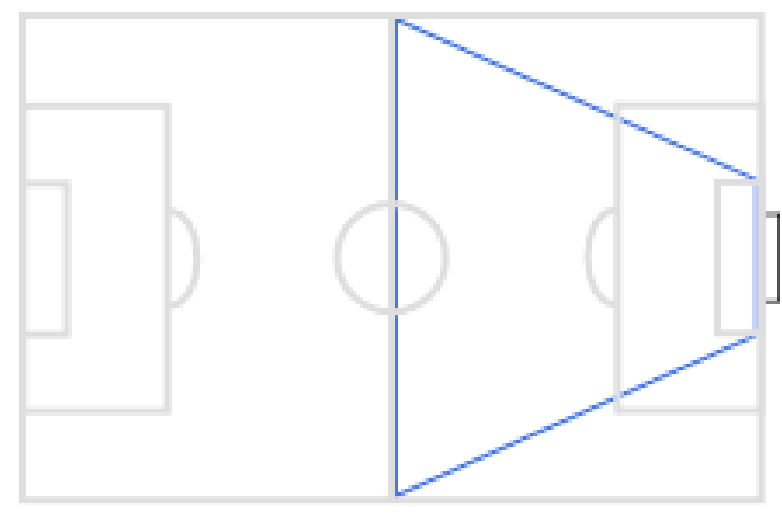

Figure 1. L'espace de jeu direct

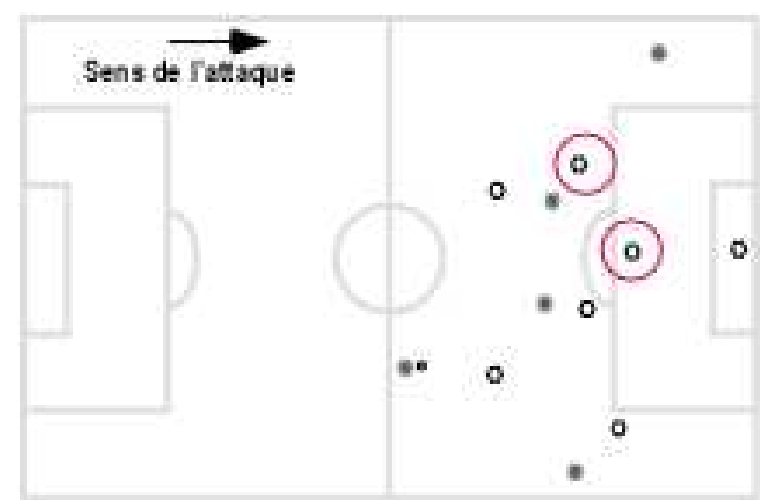

Figure 2. Hors-jeu de position ou pas

A l'origine, le hors-jeu visait à rétablir l'équilibre attaque / défense. De fait, cela a surtout contribué à limiter l'occupation de l'espace et à proposer une solution tactique, au front du ballon, à la défense. Sans la règle du hors-jeu, le football serait peut être un jeu sans échange de balle, rempli de coups de pieds vers l'avant ou de passes longues d'un bout à l'autre du terrain. En empêchant toute position trop avancée, la règle met l'accent sur la conduite de balle et la passe, plutôt que sur la longueur des coups de pieds. Cela favorise le jeu collectif, qui, à son tour, encourage de passer rapidement d'un côté du champ à l'autre mais comprime l'action sur une plus petite superficie du terrain - habituellement environ 30 ou 40 mètres de long. Le résultat final est que tous les joueurs doivent rester proches de l'action, ce qui permet à chacun de participer aux différentes configurations $\mathrm{du}$ jeu, et dans le même temps rétrécit l'espace de jeu effectif.

Toutefois, cela prive la règle du hors-jeu d'une grande partie de sa crédibilité. Si l'on veut faciliter l'attaque, il suffit d'obliger les défenseurs à défendre debout afin de protéger l'intégrité physique du porteur de balle, de favoriser les prises de risques des attaquants et de faire évoluer le score plus facilement. Si les lois du jeu devaient être amendées, il pourrait être utile de s'inspirer du futsal qui limite l'usage du tacle pour protéger les actions de joueurs en attaque. Pourquoi ne pas introduire cette règle afin de faciliter l'évolution des attaquants et de provoquer l'apparition de nouvelles techniques défensives (Duprat, 2005) intelligentes, appropriées? Cette limitation dans l'usage du tacle entraînerait moins d'agressions, moins de blessures, moins de coupures du jeu avec intervention des soigneurs et donc une plus grande continuité et fluidité du jeu. Cela serait sans doute préférable pour le jeu, le spectacle et sûrement moins coûteux en temps que l'usage de la vidéo. 


\section{eJRIEPS 32 avril 2014}

Par ailleurs, cette règle du hors-jeu n'est pas facile à appliquer et fait sans cesse l'objet de contestation. L'arbitre, juge suprême, dispose de 300 millisecondes en moyenne pour prendre sa décision, face à des spectateurs mal placés et loin de l'action mais pour qui, de toute façon, il y a hors-jeu. Autre avatar, les experts de la télévision qui, avec le recul, la technologie et le temps nécessaire, rencontrent quand même parfois d'énormes difficultés pour décider s'il y a hors-jeu ou pas, voire tout simplement déterminer quand le ballon a été effectivement joué ... Hors, ces même experts montrent parfois un incident de jeu au ralenti et sous plusieurs angles pour déclarer que l'arbitre central ou le juge de ligne s'est trompé parce que le joueur était, en fait, légèrement hors-jeu et qu'il n'a pas été signalé.

Au plan international, la discussion entre pro hors-jeu et ceux qui prônent sa disparition alimente un débat sans fin. Pour les premiers le hors-jeu doit rester en l'état, insistant sur le respect des traditions. C'est oublier que le hors-jeu est une règle qui a été créée et utilisée à une époque où il y avait une approche de «gentlemen » voire «bourgeoise » dans les sports. Un « vrai » sportif n'aurait jamais osé gagner un point tandis que ses adversaires n'étaient pas prêts à s'opposer à lui (Morris, 1981). Avec ce cadre de référence à l'esprit, le hors-jeu avait, effectivement un sens. Maintenant, les équipes du «football business» ne se soucient pas de «tradition sportive », elles ne se soucient, bien souvent, que de gagner. Cela ne cadre plus avec la tradition sportive et l'esprit du hors-jeu où nul ne doit s'arroger un avantage immérité. Au contraire, un joueur qui tire parti de tout voire qui triche discrètement passe pour un « joueur qui a de l'expérience ». Les rapports des individus aux règles peuvent être étudiés dans le type de rapports établis aux normes, mais également dans la façon dont les individus construisent leur propre règlement en jouant de la règle.

Le football amateur peut-il s'accommoder d'une suppression de la règle du hors-jeu ? Sans doute plus facilement car le rythme du jeu n'est pas le même et une occupation statique du terrain est souvent de mise. Pas de télévision, pas d'arbitre de touche « surveillé », on en revient à des conditions dignes des débuts du jeu. Les pros hors-jeu insistent sur le fait que supprimer la règle serait un bouleversement qui introduirait un coin dans l'une des parties fondamentales des règles du jeu. « La règle évite qu'un attaquant attende tranquillement devant le but adverse que ses coéquipiers lui envoient le ballon » est-il affirmé couramment. Avec des rapports d'opposition de plus en plus difficiles à gérer, se priver constamment d'un joueur est-il possible ? La suppression de la règle entraînerait, sans aucun doute, une évolution importante des tâches du gardien de but 


\section{eJRIEPS 32 avril 2014}

accentuant son rôle de « dernier défenseur » mais en ayant l'avantage de pouvoir se servir de ses mains.

Pour ses adeptes « conservateurs », la loi du hors-jeu est d'une très grande richesse dans les possibilités qu'elle offre au niveau de la diversité tactique et stratégique. Elle est source d'intelligence. Elle est aussi source de complexité pour les différents acteurs en ce qu'elle enrichit les stratégies à mettre en œuvre pour l'utiliser en défense et pour l'éviter en attaque. Comme dans une partie d'échec ou dans les activités de duels et d'opposition, le jeu d'action-réaction-action offre une grande liberté d'agir et rend la pratique encore plus attrayante. Cette règle est un élément signifiant. Les opportunités qu'elle offre et l'intelligence qu'elle suppose en soulignent toute la richesse. Elle élargit la palette des possibles au niveau du jeu et oblige les joueurs à inscrire leurs actions dans un processus pertinent. Réguler la vitesse de sa course ou modifier sa trajectoire pour éviter de dépasser la ligne fatidique avant la passe participe à l'apprentissage de déplacements intelligents. Monter sur le porteur de balle pour «forcer» la passe et provoquer une position de hors-jeu de l'attaquant délaissé est un apport tactique pertinent. Organiser cette même action avec l'aide des partenaires pour répondre au choix de pénétration en dribble de l'attaquant s'inscrit dans l'apprentissage de la coopération en défense. Coordonner les courses entre partenaires pour que le joueur en position de hors-jeu revienne, sans idée de repartir, en assurant à son tour la couverture d'un partenaire qui pénètre de derrière, entre dans la collaboration offensive. Utiliser la remise en touche rapide alors que la défense n'est pas en place et que l'on peut se mouvoir dans son dos. Surprendre les attaquants lors d'un coup franc en notre (leur) défaveur par une montée rapide lors de la prise d'élan du tireur. Baser son jeu offensif sur une remontée rapide du terrain et une reconquête très haute dans le terrain adverse en s'appuyant sur le hors-jeu. Cela ne représente-t-il pas des exemples d'enrichissement de la pratique?

\section{Conclusion}

L'objectif de cet article était d'examiner le règlement du football, son évolution et son application et de questionner la nécessité de la règle de la loi 11 du football, compte tenu de l'évolution des rapports d'opposition. L'analyse effectuée montre que les règles du jeu sont centrales et créent les conditions du bon déroulement des rencontres sportives quel que soit le lieu où elles se déroulent et le niveau auquel elles se pratiquent. En général, la règle sportive peut être envisagée comme une construction collective permanente qui établit des relations dialectiques avec l'évolution du jeu. Ces règles sportives sont souvent 


\section{eJRIEPS 32 avril 2014}

pensées comme figées pourtant elles vivent et se transforment au cours du temps. Ainsi, l'évolution des règles sportives témoigne de la dynamique inventive de l'homme (Léziart, Gabagno, Loquet, \& Trohel, 2012). Que les réalisations des joueurs dépendent directement des règles qui régissent ce jeu semble une réalité incontournable. II faut pourtant, dès qu'on y regarde de plus près, constater que le jeu sportif collectif de petit ou grand terrain est bien produit à partir des règles mais que, cependant, il constitue une réalité à part, à la fois dépendante et indépendante du règlement. Le jeu lui-même possède une vie propre, il se transforme petit à petit avec la pratique des joueurs au fil du temps.

Concernant le football, une des questions essentielles discutée dans ce texte, est bien de savoir si cette règle du hors-jeu doit être maintenue compte tenu de l'évolution du jeu et de l'environnement ou s'il est nécessaire de la modifier. Dans ce dernier cas, quelles modifications proposer pour le hors-jeu ? Le recours à la vidéo est-il la solution ? Peut-on imaginer ne la conserver qu'au plus haut niveau par exemple, ou la supprimer pour tout le monde ? Ne pourrait-on pas aussi imaginer que cette règle ne puisse s'appliquer que dans la surface de réparation ou dans le dernier quart du terrain comme au « foot à 7 »? Cela éviterait très certainement beaucoup de palabres et d'incidents sur l'ensemble des terrains où se joue le football amateur le dimanche après-midi et conséquence directe, un jeu beaucoup plus « apaisé ».

\section{Bibliographie}

Andrieu, B. (Ed.). (2013). Ethique du sport. Paris : l'Age d'homme

Brackenridge, C. (1979). Games : Classification and analysis. Conférence présentée aux Kirkless Teachers, le 02 mai 1979.

Daryl, P. (1894). Jeux de balle et de ballon. Paris : Imprimeries réunies.

Deleplace, R. (1966). Le rugby. Paris : Colin-Bourrelier.

Deleplace, R. (1979). Rugby de mouvement-Rugby total. Paris: Éducation Physique et Sport.

Dosseville, F., \& Laborde, S. (2011). Les facettes de l'arbitrage : Recherches et problématiques actuelles. Paris : Editions Publibook.

Duprat, E. (2005). Approche technologique de la récupération du ballon lors de la phase défensive en football, contribution à l'élaboration de contenus de formations innovants. Thèse de Doctorat (non publiée). École Normale Supérieure de Cachan. 


\section{eJRIEPS 32 avril 2014}

Fuss, F., Subic, A., \& Ujihashi, S. (Eds.) (2007). The Impact of Technology on Sport II. London : Taylor \& Francis.

Gréhaigne, J.-F. \& Marle, P. (2014) L'activité des joueurs en sport collectif : l'exemple du football. In J.-F. Gréhaigne, (Ed.). L'intelligence tactique. Des perceptions aux décisions tactiques en sports collectifs (pp. 221-242). Besançon: Presses de l'Université de Franche-Comté.

Gréhaigne, J.-F., Meunier, J.-N., \& Vigneron, C. (2007). Ethique, arbitrage, règles et sport collectif. In J.F. Gréhaigne (Ed.) Configurations du jeu. Débat d'idées et apprentissage du football et des sports collectifs (pp. 123-132). Besançon: Presses de l'Université de Franche-Comté.

Kssis, N., \& Couvidou, P. (2013). Petite histoire de l'arbitrage en foot. Sport et plein air, 574, 32-33.

Lipovetsky, G. (1983). L'ère du vide. Essais sur l'individualisme contemporain. Paris : Gallimard.

Léziart, Y., Gabagno, G., Loquet, M., \& Trohel, J. (Eds.). (2012). La règle sportive. Presses universitaires de Bordeaux.

Morris, D. (1981). The soccer tribe. London : Jonathan Cape.

Offside (nd) From Wikipedia, the free encyclopedia. http://en.wikipedia.org/wiki/Offside

Teneze, L. (2011). Histoire du football. Le Board et l'analyse des transformations des lois du jeu. Thèse de Doctorat (non publiée). Université Paris V.

Tunmer, A.A., \& Fraysse, E. (1904). Football (Association). Paris : Colin. 